\title{
Engineering domain configurations for enhanced piezoelectricity in barium titanate single crystals
}

\author{
J. J. Liu and Y. C. Zhou \\ Key Laboratory of Advanced Materials and Rheological Properties of Ministry of Education, \\ Faculty of Materials and Optoelectronic Physics, Xiangtan University, Xiangtan, Hunan 411105, China \\ A. K. Soh
Department of Mechanical Engineering, University of Hong Kong, Hong Kong, China
J. Y. Li ${ }^{\text {a) }}$
Department of Mechanical Engineering, University of Washington, Seattle, Washington 98195-2600
}

(Received 17 August 2005; accepted 21 November 2005; published online 20 January 2006)

In this letter, we propose a mechanism for enhanced piezoelectricity that takes advantage of both intrinsic crystalline anisotropy of ferroelectric crystal and extrinsic $90^{\circ}$ domain switching of ferroelectric variant. The domain configurations we consider here are not clamped, and thus allow domain switching driven by the competition between external electric field and internal depolarization field. The intrinsic and extrinsic piezoelectric responses of barium titanate single crystals under different crystallographic orientations are calculated using an energy minimization theory, where it is observed that the piezoelectric coefficient is significantly enhanced by the $90^{\circ}$ domain switching, especially under the small field measurement where the domain wall movement is reversible, which is consistent with recent experimental observations. The optimal crystallographic orientation is also identified. (C) 2006 American Institute of Physics.

[DOI: $10.1063 / 1.2166480$ ]

Piezoelectric materials with large actuation strains and high electromechanical coupling factors have a wide range of applications in sensors, actuators, and transducers, ${ }^{1}$ which has stimulated a large volume of studies in the last decade searching for new piezoelectric materials or novel actuation mechanisms. These works can mainly be classified into two groups, one based on crystalline anisotropy by poling a ferroelectric crystal along a nonpolar axis, where piezoelectric coefficients larger than $2000 \mathrm{pC} / \mathrm{N}$ have been obtained in $\mathrm{Pb}\left(\mathrm{Mg}_{1 / 3} \mathrm{Nb}_{2 / 3}\right) \mathrm{O}_{3}-\mathrm{PbTiO}_{3}$ (PMN-PT) and $\mathrm{Pb}\left(\mathrm{Zn}_{1 / 3} \mathrm{Nb}_{2 / 3}\right) \mathrm{O}_{3}-\mathrm{PbTiO}_{3}$ (PZN-PT) crystals; ${ }^{2-5}$ and the other based on $90^{\circ}$ domain switching by introducing competitions among ferroelectric variants using various biasing fields, where actuation strains as high as $1 \%$ have been demonstrated in $\mathrm{BaTiO}_{3}$ crystals. $^{6-8}$ In this letter, we propose a mechanism that combines these two approaches for enhanced piezoelectricity, and use it to explain the ultrahigh piezoelectric response recently observed in a (270) cut barium titanate. ${ }^{9}$

While the coexistence of variants in a ferroelectric crystal poled along a nonpolar axis is commonly referred to as "engineered domain configuration", ${ }^{2-5}$ such as rhombohedral crystals PMN-PT and PZN-PT poled along [001] axis, and tetragonal crystal barium titanate poled along [111] axis, these domains are often clamped and the enhanced piezoelectricity observed is more related to the crystalline anisotropy than a particular domain configuration. ${ }^{5,10-13}$ On the other hand, the large actuation strain obtained through $90^{\circ}$ domain switching in $\mathrm{BaTiO}_{3}$ crystal requires a biasing field either by using an external mechanical loading device, ${ }^{6,7}$ or

\footnotetext{
${ }^{a)}$ Author to whom correspondence should be addressed; also with: Key Laboratory of Advanced Materials and Rheological Properties of Ministry of Education, Faculty of Materials \& Optoelectronic Physics, Xiangtan University, China; electronic mail: jjli2@unl.edu
}

through internal defect mediation of domain walls, ${ }^{8}$ and thus is not very convenient to use. Our proposed mechanism will overcome these difficulties while maintain the advantage of both approaches.

We seek to take advantage of the crystalline anisotropy of ferroelectric crystals by poling them along a nonpolar axis, and thus enhance their intrinsic piezoelectric response, while at the same time induce $90^{\circ}$ domain switching using the competition between the external electric field and internal depolarization field, and thus enhance their extrinsic piezoelectric response without external mechanical loading device or internal defect mediation. To accomplish this, let us consider a tetragonal crystal, such as $\mathrm{BaTiO}_{3}$, cut along $\left(n_{1} n_{2} 0\right)$ surface and poled along $\left[n_{1} n_{2} 0\right]$ axis, as shown in Fig. 1. The domain configuration of the crystal can then be determined by minimizing the potential energy of the system, ${ }^{14,15}$

$$
F(\boldsymbol{\varepsilon}, \mathbf{p})=\int_{\Omega}\left[W(\boldsymbol{\varepsilon}, \mathbf{p})-\mathbf{E}_{0} \cdot \mathbf{p}\right] d \mathbf{x}+\frac{\kappa}{2} \int_{\mathrm{R}^{3}}|\nabla \phi|^{2} d \mathbf{x},
$$

where we have ignored the domain-wall energy, which is justified for large specimen. ${ }^{16}$ In the potential energy, the first

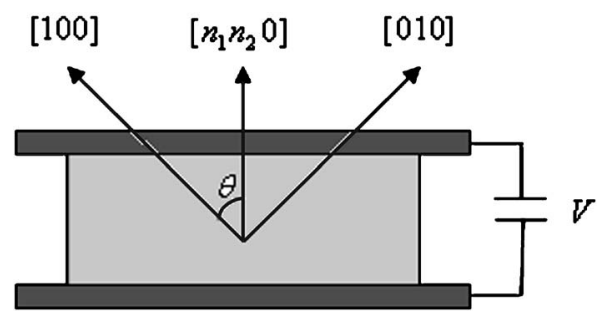

FIG. 1. A tetragonal crystal cut along $\left(n_{1} n_{2} 0\right)$ surface and poled along $\left[n_{1} n_{2} 0\right]$ axis. 
term is the stored energy density of the ferroelectric which depends on the state variables, strain $\varepsilon$ and polarization $\mathbf{p}$. It encodes the information that the ferroelectric prefers certain states of transformation strain and polarization, under which $W(\boldsymbol{\varepsilon}, \mathbf{p})=0 .^{5}$ For tetragonal crystal, they are

$$
\begin{array}{ll}
\boldsymbol{\varepsilon}^{( \pm 1)}=\left[\begin{array}{lll}
\beta & 0 & 0 \\
0 & \alpha & 0 \\
0 & 0 & \alpha
\end{array}\right], & \mathbf{p}^{( \pm 1)}= \pm\left[\begin{array}{c}
p_{t} \\
0 \\
0
\end{array}\right], \\
\boldsymbol{\varepsilon}^{( \pm 2)}=\left[\begin{array}{lll}
\alpha & 0 & 0 \\
0 & \beta & 0 \\
0 & 0 & \alpha
\end{array}\right], & \mathbf{p}^{( \pm 2)}= \pm\left[\begin{array}{c}
0 \\
p_{t} \\
0
\end{array}\right], \\
\boldsymbol{\varepsilon}^{( \pm 3)}=\left[\begin{array}{lll}
\alpha & 0 & 0 \\
0 & \alpha & 0 \\
0 & 0 & \beta
\end{array}\right], & \quad \mathbf{p}^{( \pm 3)}= \pm\left[\begin{array}{c}
0 \\
0 \\
p_{t}
\end{array}\right],
\end{array}
$$

given in the cubic coordinate system with $x_{1}\left\|[100], x_{2}\right\|[010]$, and $x_{3} \|[001]$. The second term is the interaction energy with the applied electric field $\mathbf{E}_{0}$, which tends to align the polarization toward $\mathbf{E}_{0}$. Under a uniform external electric field $E_{0}$ along $\left[n_{1} n_{2} 0\right]$ axis, only variants 1 and 2 are energetically preferred, leading to the coexistence of variants 1 and 2, such that the interaction energy becomes $-E_{0}\left\langle p_{\|}\right\rangle=-E_{0} p_{t}\left(f_{1} \cos \theta\right.$ $\left.+f_{2} \sin \theta\right)$, where $\left\langle p_{\|}\right\rangle$is the volume averaged out-of-plane polarization component parallel to $\left[n_{1} n_{2} 0\right], \theta$ is the angle between [100] and $\left[n_{1} n_{2} 0\right]$, and $f_{1}$ and $f_{2}=1-f_{1}$ are volume fraction of variants 1 and 2, respectively. The last term is the depolarization energy due to the electric field generated by the polarization distribution in the ferroelectric, where the permittivity of the ferroelectric crystal, $\kappa$, rather than that of the free space, is used to account for the polarizability of the ferroelectric crystal under an electric field. Under the flatplate configuration of Fig. 1, the depolarization energy is evaluated as $\frac{1}{2 \kappa}\left\langle p_{\perp}\right\rangle^{2}=\frac{p_{t}^{2}}{2 \kappa}\left(f_{1} \sin \theta-f_{2} \cos \theta\right)^{2}$, where we have ignored the geometric dependence, and $\left\langle p_{\perp}\right\rangle$ is the volume averaged in-plane polarization component parallel to $\left(n_{1} n_{2} 0\right)$. As such, the potential energy per unit volume is

$$
F=-E_{0} p_{t}\left(f_{1} \cos \theta+f_{2} \sin \theta\right)+\frac{p_{t}^{2}}{2 \kappa}\left(f_{1} \sin \theta-f_{2} \cos \theta\right)^{2},
$$

from which the volume fractions $f_{1}$ of variants 1 can be derived;

$$
f_{1}=\frac{\kappa E_{0}(\cos \theta-\sin \theta)}{p_{t}(\sin \theta+\cos \theta)^{2}}+\frac{\cos \theta}{\sin \theta+\cos \theta} .
$$

Clearly, for a crystal with given orientation, the volume fraction of variant 1 is determined by the applied electric field. The domain configuration in a ferroelectric crystal having two variants coexisting can then be constructed by a rankone lamination, ${ }^{17}$ and its intrinsic piezoelectric coefficient can be calculated using a procedure developed in Ref. 5, with the volume fraction $f_{1}$ and the electromechanical moduli of single-domain single crystal serving as input. When there is no external electric field applied, the intrinsic piezoelectric coefficient $d_{33}$ as a function of $\theta$ measured along $\left[n_{1} n_{2} 0\right]$ axis is shown in Fig. 2, and it is observed that the curve is symmetric with respect to $\theta=45^{\circ}$, or [110] axis, where the piezoelectric coefficient $d_{33}=219.5 \mathrm{pC} / \mathrm{N}$ is maxi-

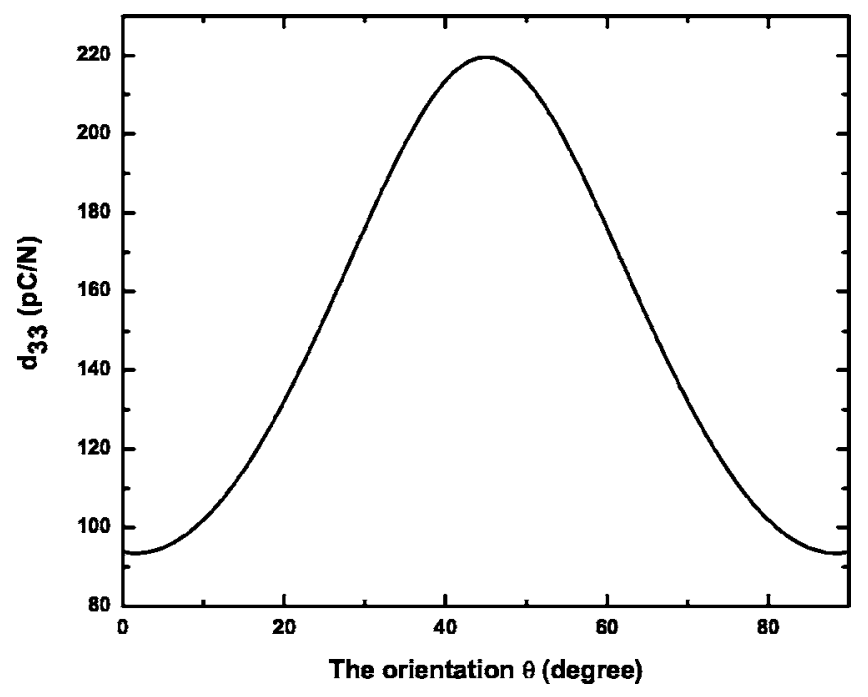

FIG. 2. The electromechanical strain as function of the applied electric field $E_{0}$ at different $\theta$.

mum, more than $100 \%$ higher than that of the single-domain single crystal, consistent with our previous finding. ${ }^{17}$

The piezoelectric coefficient calculated here is intrinsic at zero field, and it is more informative to consider the total strain induced by the applied electric field, which consists of two parts; one due to the intrinsic piezoelectricity, and the other due to the change of volume fraction $f_{1}$ under the applied electric field, the so-called transformation strain. Using $E_{0}=0$ as the reference, the total strain can be obtained from

$$
\epsilon_{33}=d_{33} E_{0}+\left\langle\varepsilon_{33}^{T}\left(E_{0}\right)\right\rangle-\left\langle\varepsilon_{33}^{T}(0)\right\rangle,
$$

where $\left\langle\varepsilon_{33}^{T}\right\rangle=f_{1} \varepsilon_{33}^{(1)}+f_{2} \varepsilon_{33}^{(2)}$ is the volume averaged transformation strain along $\left[n_{1} n_{2} 0\right]$ axis. The total strain induced by the applied electric field $E_{0}$ at different $\theta$ is shown in Fig. 3, where it is observed that for $0<\theta \leqslant 7.5^{\circ}$, the curves have two linear segments; the first due to the change of volume fraction $f_{1}$, or $90^{\circ}$ domain switching, which has larger slope, while the second due to the intrinsic piezoelectricity at single-domain state, or after saturation. Similar change of slope has recently been observed in a (270) cut barium titanate crystal. ${ }^{9}$ For $10^{\circ} \leqslant \theta<45^{\circ}$, only one linear segment is observed, since the crystal has not saturated yet under the

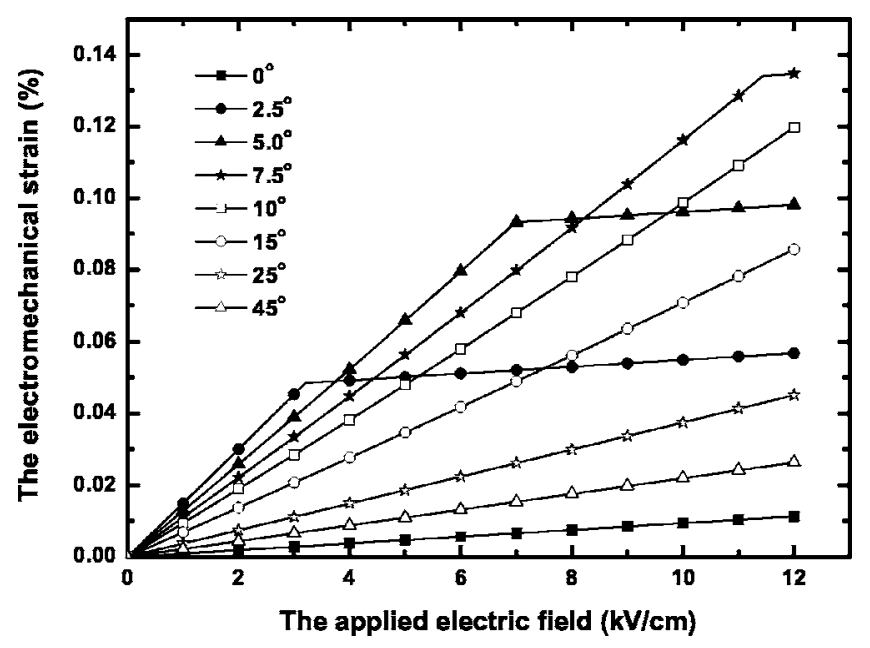

FIG. 3. The electromechanical strain as function of the applied electric field $E_{0}$ at different $\theta$. 


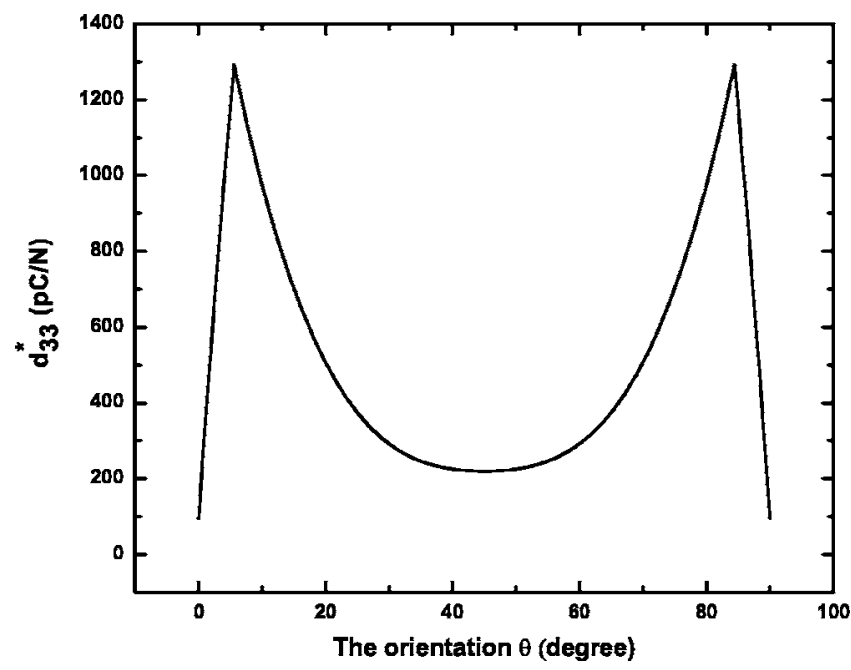

FIG. 4. The total piezoelectric coefficient $d_{33}^{*}$ as a function of $\theta$.

applied electric field $E_{0} \leqslant 12 \mathrm{kV} / \mathrm{cm}$. The total piezoelectric coefficient $d_{33}^{*}$ thus has contributions from both intrinsic and extrinsic effects, calculated from $d_{33}^{*}=\varepsilon_{33} / E_{0}$, which is shown in Fig. 4 as a function of the orientation $\theta$. It is observed that the piezoelectric coefficient $d_{33}^{*}$ is also symmetric with respect to [110] axis, increasing quickly from $\theta=0^{\circ}$, reaching maximum value around $1300 \mathrm{pC} / \mathrm{N}$ at $\theta=5^{\circ}$, and then decreasing quickly afterward, reaching minimum at $\theta$ $=45^{\circ}$. This suggests that $90^{\circ}$ domain switching can contribute significantly to the piezoelectric response, especially under a small electric-field measurement where the domainwall movement is reversible. When a large electric field is applied that saturates the ferroelectric single crystal, the $d_{33}^{*}$ then will be much smaller. Recently, an ultrahigh piezoelectric response with $d_{33}$ as high as $2000 \mathrm{pC} / \mathrm{N}$ was reported in a (270) cut barium titanate crystal, ${ }^{9}$ one order of magnitude larger than the largest intrinsic piezoelectric coefficient of $219.5 \mathrm{pC} / \mathrm{N}$. We believe that such a ultrahigh piezoelectric response is induced by $90^{\circ}$ domain switching due to the competition of external electric field and depolarization field. It is also worthwhile to notice that the $2000 \mathrm{pC} / \mathrm{N}$ value is obtained from the small field measurement using $d_{33}$ meter, while the value obtained from the strain measurement at high electric field is much smaller, also consistent with our analysis. We have to point out that at [720], or $\theta=16^{\circ}$, our calculated $d_{33}^{*}$ is around $700 \mathrm{pC} / \mathrm{N}$; smaller than the experimental value, yet still much larger than the intrinsic one. This may be caused by switching of residual variant 3 in the experiment, which we have not considered.

In summary, we proposed a mechanism that takes advantages of both intrinsic crystalline anisotropy of ferroelectric crystal and the extrinsic switching of ferroelectric variants. Unlike the engineered domain configuration previously reported, the domain configuration considered here is not clamped; allowing contribution from $90^{\circ}$ domain switching. In addition, the biasing field for $90^{\circ}$ domain switching proposed here is from the internal depolarization field, not requiring an external mechanical device or manipulation of internal defect structure. This mechanism can also be used to explain the ultrahigh piezoelectric response recently reported in a (270) cut barium titanate.

The work was partially supported by U.S. National Science Foundation (No. CMS-0415261), Natural Science Foundation of China (Approval No. 10572124), and Research Grants Council of the Hong Kong Special Administrative Region, China (Project No. HKU 7086/02E). The authors also acknowledge Furong Scholars Programme of Hunan Province, China and the Undergraduate Innovation Fund of Xiangtan University, China.

${ }^{1}$ K. Bhattacharya and G. Ravichandran, Acta Mater. 51, 5941 (2003).

${ }^{2}$ S. E. Park and T. R. Shrout, J. Appl. Phys. 82, 1804 (1997).

${ }^{3}$ S. Wada, S. Suzuki, T. Noma, T. Suzuki, M. Osada, M. Kakihana, S. E. Park, L. E. Cross, and T. Shrout, Jpn. J. Appl. Phys., Part 1 38, 5505 (1999).

${ }^{4}$ H. S. Luo, G. S. Xu, H. Q. Xu, P. C. Wang, and Z. W. Yin, Jpn. J. Appl. Phys., Part 1 39, 5581 (2000).

${ }^{5}$ J. Y. Li and D. Liu, J. Mech. Phys. Solids 52, 1719 (2004).

${ }^{6}$ E. Burcsu, G. Ravichandran, and K. Bhattacharya, Appl. Phys. Lett. 77, 1698 (2000).

${ }^{7}$ E. Burcsu, G. Ravichandran, and K. Bhattacharya, J. Mech. Phys. Solids 52, 823 (2004).

${ }^{8}$ X. Ren, Nat. Mater. 3, 91 (2004).

${ }^{9}$ R. Chu, Z. Xu, G. Li, H. Zeng, H. Yu, H. Luo, and Q. Yin, Appl. Phys. Lett. 86, 012905 (2005).

${ }^{10}$ R. Zhang, B. Jiang, and W. W. Cao, Appl. Phys. Lett. 82, 3737 (2003).

${ }^{11}$ D. Damjanovic, M. Budimir, M. Davis, and N. Setter, Appl. Phys. Lett. 83, 527 (2003).

${ }^{12}$ T. Liu and C. S. Lynch, Acta Mater. 51, 407 (2003).

${ }^{13}$ D. Liu and J. Y. Li, Appl. Phys. Lett. 84, 3930 (2004).

${ }^{14}$ Y. C. Shu and K. Bhattacharya, Philos. Mag. B 81, 2021 (2001).

${ }^{15}$ J. Y. Li, R. C. Rogan, E. Üstündag, and K. Bhattacharya, Nat. Mater. 4, 776 (2005).

${ }^{16}$ A. Desimone, Arch. Ration. Mech. Anal. 125, 99 (1993).

${ }^{17}$ D. Liu and J. Y. Li, Appl. Phys. Lett. 83, 1193 (2003). 